\title{
Preface
}

\section{Regional Anesthesiology and Acute Pain Medicine in the Era of Value-Based Health Care}

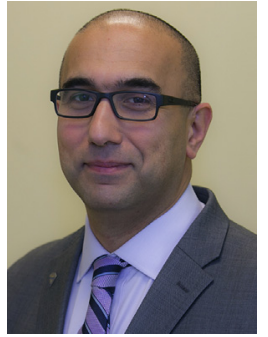

Nabil M. Elkassabany, MD, MSCE

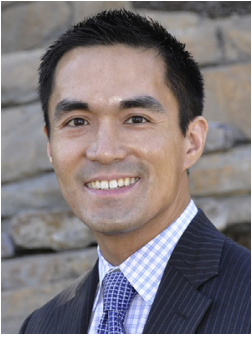

Edward R. Mariano, MD, MAS (Clinical Research) Editors

The practice of regional anesthesia has gone through a noticeable transformation. Now defined by the Accreditation Council for Graduate Medical Education (ACGME) as a subspecialty of anesthesiology, developments in regional anesthesiology and acute pain medicine offer effective alternatives to general anesthesia and form the basis of opioid-sparing multimodal analgesia for surgical patients. For the past 50 years, study after study has examined the quality of postoperative pain management resulting from peripheral nerve and neuraxial blockade, yet the results consistently show that most patients still complain of significant pain after surgery. We still have work to do.

Throughout the history of regional anesthesia research, a primary focus has been the technical aspects of performing procedures - whether needle placement in location $X$ versus location $Y$ will result in better blocks. While expertise in relevant anatomy, pharmacology, and needle skills are necessary elements for successful implementation of regional anesthesiology and acute pain medicine, these elements are not sufficient. The goals of today's regional anesthesiology and acute pain medicine specialists have gone way beyond the "block." They now have embraced the role of physician leaders and are poised to disseminate and implement new perioperative care models.

This issue of Anesthesiology Clinics is a reflection of this viewpoint. We are grateful to the series editor, Dr Fleisher, for the opportunity to present a collection of articles that reflect the evolution of regional anesthesiology and acute pain medicine. This editorial collaboration is an exciting new project for the two of us. For the past several years, we have worked together on many projects promoting the subspecialty of regional anesthesiology and acute pain medicine: developing the competency-based milestones for the newly ACGME-accredited regional anesthesiology and acute pain medicine fellowships; working together on the American Society of Regional 
Anesthesia and Pain Medicine (ASRA) communications committees and ASRA Board of Directors; developing national quality measures in pain management; collaborating on clinical research projects; and coediting a previous issue of Anesthesiology Clinics on "Orthopedic Anesthesiology." In this issue, we have carefully selected articles that highlight the value that regional anesthesia offers patients and how physicians can establish their own programs in regional anesthesiology and acute pain medicine in various practice settings. These topics include building an ambulatory perineural catheter program for pediatric patients, the use of regional anesthesia in trauma patients, and establishing enhanced recovery protocols for joint arthroplasty patients. We also cover some timely and challenging topics: managing patients on opioid replacement therapy, newer drugs on the horizon for multimodal analgesia, new frontiers and technologies in regional anesthesia, and the use of "big data" to study the most important outcomes that matter to our patients.

We are grateful for our families for their unwavering support, our authors for donating their time and expertise, and the readership of Anesthesiology Clinics. It is our hope that this final product represents an up-to-date comprehensive review of the most pressing issues pertaining to value-based regional anesthesiology and acute pain medicine and offers something for everyone.

Nabil M. Elkassabany, MD, MSCE Sections of Orthopedic and Regional Anesthesiology Department of Anesthesiology and Critical Care

Perelman School of Medicine University of Pennsylvania

3400 Spruce Street, Dulles 7th

Philadelphia, PA 19104, USA

Edward R. Mariano, MD, MAS (Clinical Research)

Department of Anesthesiology

Perioperative and Pain Medicine

Stanford University School of Medicine

Anesthesiology and Perioperative Care Service

VA Palo Alto Health Care System

3801 Miranda Avenue (112A)

Palo Alto, CA 94304, USA

E-mail addresses:

Nabil.Elkassabany@uphs.upenn.edu (N.M. Elkassabany) emariano@stanford.edu (E.R. Mariano) 